\title{
Compact planar far-field superlens based on anisotropic left-handed metamaterials
}

\author{
Nian-Hai Shen, ${ }^{1, *}$ Stavroula Foteinopoulou, ${ }^{1,2}$ Maria Kafesaki, ${ }^{1,3}$ Thomas Koschny, ${ }^{1,2}$ Ekmel Ozbay, ${ }^{4}$ \\ Eleftherios N. Economou, ${ }^{1}$ and Costas M. Soukoulis $1,2,3, \dagger$ \\ ${ }^{1}$ Institute of Electronic Structure and Lasers (IESL), Foundation of Research and Technology-Hellas (FORTH), 71110 Heraklion, Crete, \\ Greece \\ ${ }^{2}$ Ames Laboratory, U.S. DOE, and Department of Physics and Astronomy, Iowa State University, Ames, Iowa 50011, USA \\ ${ }^{3}$ Department of Materials Science and Technology, University of Crete, 71003 Heraklion, Crete, Greece \\ ${ }^{4}$ Nanotechnology Research Center, Department of Physics, and Department of Electrical and Electronics Engineering, Bilkent University, \\ Bilkent, 06800 Ankara, Turkey
}

(Received 10 July 2009; revised manuscript received 26 August 2009; published 24 September 2009)

\begin{abstract}
Pendry's perfect lens has spurred intense interest for its practical realization at visible frequencies. However, fabrication of low-loss isotropic left-handed metamaterials is a current challenge. In this work, we theoretically show that under specific conditions anisotropic metamaterial slabs can emulate Pendry's perfect-lens phenomenon on a plane. Geometric optics leads to a new lens formula for this special anisotropic metamaterial superlens, which allows significant shrinkage of the metamaterial slab thickness for a certain range of far-field operation. Conversely, such anisotropic metamaterial superlens with the same thickness as its isotropic analog can operate for much larger distances between object and lens. We present numerical simulations which confirm our theoretical calculations. In particular, we find subdiffraction focusing that rivals the perfect isotropic negative-index metamaterial lens performance and obeys the new lens formula as predicted. In addition, we demonstrate that it is possible to attain far-field superfocusing with a metamaterial slab as thin as half the free-space wavelength. We believe this work will inspire new anisotropic metamaterial designs and opens a promising route for the realization of compact far-field superlenses in the visible regime.
\end{abstract}

DOI: 10.1103/PhysRevB.80.115123

PACS number(s): 78.20.Ci, 42.30.Wb, 42.25.Bs

\section{INTRODUCTION}

Pendry envisioned the existence of a "perfect lens" in $2000,{ }^{1}$ which would resolve beyond the diffraction limit. Such perfect lens is essentially a flat slab made of the Veselago negative-index metamaterial (NIM) (Ref. 2) having both permittivity and permeability simultaneously negative and equal to -1 . The basic mechanism which leads to the super-resolving power of the perfect lens is the reconstruction of all wave vectors emitted from the source, both radiative (far field) and dark (near field). The NIM with permittivity and permeability simultaneously equal to -1 causes a beam to totally refract with a negative angle equal in magnitude to the angle of incidence from air. As a result, all far-field Fourier components of a point source meet at the same point inside such metamaterial slab and cross again after refracting at the second slab interface with no loss of intensity. The near-field source components decay in air but are exactly compensated by exponential increase in their intensity inside the metamaterial slab. ${ }^{1,3}$ Consequently, an image produced by the perfect lens would contain as much detail as the object. With geometric ray tracing it can be easily shown that the distance between the source and the first slab interface and the distance between the image and the second slab interface add up exactly to the thickness of the perfect-lens slab. The latter relation is widely known as the perfect-lens formula.

Pendry's pioneering work motivated an increasing number of efforts to bring the existence of the Veselago NIM out of the realm of fiction. Effective NIMs based on different designs have been proposed and realized by various groups. ${ }^{4-6}$ Initial microwave NIM designs paved the way for fabrication of NIMs in THz and near-IR frequencies with fishnet metamaterials being realized all the way up to the visible spectrum. ${ }^{7}$ Although recent progress in new connected metamaterial designs ${ }^{8}$ and new fabrication techniques ${ }^{9}$ give promise for the realization of threedimensional isotropic NIMs, such goal has not been accomplished to this date.

Therefore, many research investigations focused on whether it would be possible to achieve subdiffraction focusing by maintaining only some of the features of Pendry's perfect lens. One explored avenue was to use photonic crystal (PC) media that emulate the refractive properties of the Veselago medium with refractive index, $n$, equal to $-1 .^{10,11}$ Indeed, subdiffraction focusing was reported in these type of photonic crystals, ${ }^{12,13}$ which emanates from the refocusing of only all far-field source components in a manner analogous to the effect in the Veselago medium with $n=-1$. These superlenses operate in the far field but their performance is restricted due to impedance mismatch losses at the air-PC interface. Then, theoretical ${ }^{14}$ and experimental ${ }^{15,16}$ works demonstrated that it is possible to achieve super-resolution by reconstructing only the dark components of the source. The latter is enabled by the coupling of surface-plasmon polaritons (SPPs) at each interface of a thin metal slab. However, unavoidably this type of superfocusing is restricted in the near-field zone only.

More recently, another path to transfer the dark components of the source has been proposed. Let's consider a uniaxial effective either electric ${ }^{17,18}$ or magnetic ${ }^{19,20}$ metamaterial where the permittivity or permeability, respectively, along the optical axis is opposite in sign to the permittivities and permeabilities in the other two principal directions. An electromagnetic (EM) wave propagating in such medium is 
subject to a hyperbolic dispersion relations and is characterized by conventional forward (not backward) type of propagation. With proper choice of the interface of a slab made from such material, this type of hyperbolic dispersion relation causes all dark components of the source to convert into propagating modes within the structure and so they transfer to the other side of the slab. The radiative source components get negatively refracted with a small angle, which causes a collimatedlike beam transfer within the structure. The combined effect leads to a pseudofocusing ${ }^{17-20}$ with subwavelength but not subdiffraction resolution. The operation of such lens is restricted to the near or close far field (on the order of a wavelength) zone. It was also demonstrated that such metamaterials with hyperbolic dispersion relation in a semicylindrical shape can lead to significant separation of subdiffraction spaced sources ${ }^{21}$ or possess magnifying properties. $^{22-24}$ Furthermore, subdiffraction resolution with one-dimensional metamaterials has been reported experimentally at microwaves ${ }^{25,26}$ but the mechanism leading to such phenomenon has not been analyzed.

Without a doubt, there have been a plethora of superlensing works which utilize only part or some modifications of Pendry's perfect-lens mechanism in order to meet the mandates of practicality. In this work, we investigate whether it is possible to observe the full perfect-lens phenomenon with easier attainable anisotropic metamaterials. Typical metamaterials that have been realized in visible frequencies are one dimensional ${ }^{4,6}$ as their magnetic resonance is excited only for a single-incident wave polarization and propagation direction (anisotropic metamaterial ${ }^{27}$ ). Furthermore, we explore whether it is possible to achieve a lens with far-field operation while keeping its thickness small. Losses are ubiquitous in practical metamaterials. Therefore, the possibility of a thin lens would have tremendous impact in the realization of a high-quality image. In the following, we present our proposal of a thin anisotropic metamaterial slab which demonstrates Pendry's perfect-lens phenomenon on a plane. We believe our theoretical analysis will spur investigations for new anisotropic metamaterial designs for superfocusing in the visible spectrum.

In Sec. II, under lossless assumption for convenience, we search theoretically for the specific necessary conditions, which an anisotropic metamaterial must satisfy in order to obtain Pendry's perfect-lens phenomenon. In Sec. III, we obtain with geometric optics a new lens formula for our proposed structure. We discuss why this new lens formula signifies the possibility to obtain a thin yet far-field superlens. In Sec. IV, we present numerical simulations which support our theoretical claims and which manifest a performance for our proposed lens on a par with the isotropic perfect-lens analog. Finally, in Sec. V we present our conclusions.

\section{SEARCHING FOR PERFECT-LENS PROPERTIES IN ANISOTROPIC METAMATERIALS}

Suppose we have an EM wave coming from an isotropic right-handed medium (RHM) with permittivity $\varepsilon_{1}>0$ and permeability $\mu_{1}>0$, on a medium which is a lossless anisotropic metamaterial with permittivity and permeability of the form

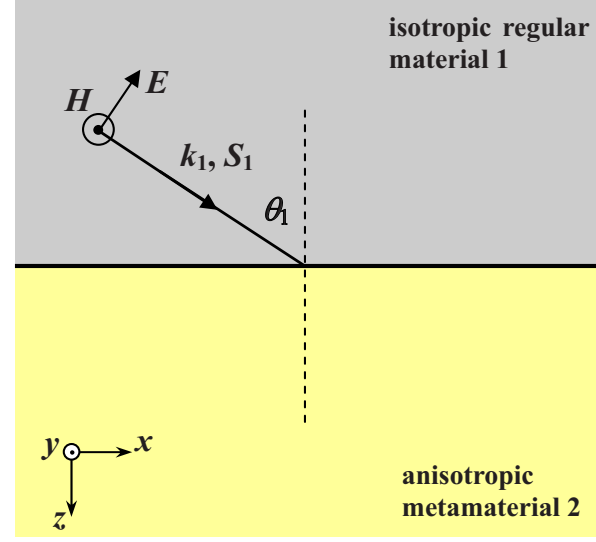

FIG. 1. (Color online) A p-polarized EM wave (magnetic field $\boldsymbol{H}$ perpendicular to the plane of incidence) comes from an isotropic medium (material 1) at oblique incidence with angle $\theta_{1}$ onto an anisotropic metamaterial (material 2). $\boldsymbol{k}_{\mathbf{1}}$ and $\boldsymbol{S}_{\mathbf{1}}$ are the wave vector and Poynting vector of the incident beam, respectively.

$$
\overline{\bar{\varepsilon}}_{2}=\left[\begin{array}{ccc}
\varepsilon_{2 x} & 0 & 0 \\
0 & \varepsilon_{2 y} & 0 \\
0 & 0 & \varepsilon_{2 z}
\end{array}\right], \quad \overline{\bar{\mu}}_{2}=\left[\begin{array}{ccc}
\mu_{2 x} & 0 & 0 \\
0 & \mu_{2 y} & 0 \\
0 & 0 & \mu_{2 z}
\end{array}\right] .
$$

We choose an incoming $p$-polarized wave and the $x z$ plane as the plane of incidence (see Fig. 1). So the components of the wave vector $\boldsymbol{k}_{\boldsymbol{i}}$, electric field vector $\boldsymbol{E}_{\boldsymbol{i}}$, and magnetic field vector $\boldsymbol{H}_{\boldsymbol{i}}$ in coordinate space would be $\left(k_{i x}, 0, k_{i z}\right)$, $\left(E_{i x}, 0, E_{i z}\right)$, and $\left(0, H_{i y}, 0\right)$ respectively, with $i=1,2$ corresponding to materials 1 and 2 .

The EM boundary conditions mandate the preservation of the magnetic fields direction inside the anisotropic metamaterial and in addition require that the parallel component of the wave vector be conserved. Thus

$$
k_{1 x}^{2}=k_{2 x}^{2}=k_{x}^{2}=k_{0}^{2} \varepsilon_{1} \mu_{1} \sin ^{2} \theta_{1},
$$

with $k_{0}=\omega / c$ being the free-space wave number and $\theta_{1}$ the angle of incidence. Moreover, from Maxwell's equations for general electric and magnetic anisotropic media, ${ }^{28,29}$ and for the specific illumination conditions given in Fig. 1, we obtain the following dispersion relation for an EM wave propagating inside the anisotropic metamaterial:

$$
\frac{k_{x}^{2}}{\varepsilon_{2 z} \mu_{2 y}}+\frac{k_{2 z}^{2}}{\varepsilon_{2 x} \mu_{2 y}}=k_{0}^{2} \text {. }
$$

Inside the isotropic material we have the following familiar expression for the dispersion relation:

$$
k_{x}^{2}+k_{1 z}^{2}=\varepsilon_{1} \mu_{1} k_{0}^{2} .
$$

These dispersion relations can be represented graphically on the $x z$ wave-vector plane as contours for different fixed frequencies. These contours are generally known as equifrequency contours (EFCs). ${ }^{11}$ Evidently, the EFCs in the isotropic medium are circles. On the other hand, Eq. (3) implies that the EFCs in the anisotropic metamaterial can be hyper- 
bolas or ellipses depending on the specific values of the permittivities in the $x z$ plane, $\varepsilon_{2 x}$ and $\varepsilon_{2 z}$, and the permeability in the $y$ direction, $\mu_{2 y}$.

Let's reiterate what are all the key ingredients of a metamaterial to behave as a perfect lens according to Pendry's proposal: (i) the metamaterial must be reflectionless for all angle of incidence so all energy of the source is transported through the slab. (ii) EM waves at oblique incidence with angle $\theta_{1}$, must refract negatively at the interface of the metamaterial with an angle $\theta_{2}$ so that $\tan \theta_{2} / \tan \theta_{1}$ is independent of the incident angle $\theta_{1}$. This ensures that all beams corresponding to the spatial spectral decomposition of a pointlike source meet up at the same point inside the metamaterial. (iii) Ramakrishna ${ }^{3}$ showed also that Pendry's perfect-lens slab possesses dispersionless SPPs, i.e., SPPs which exist for any frequency and lateral wave vector. He demonstrated that it is the coupling of these SPPs at each side of the slab which enables the exponential growth of the near-field components inside the lens material, which compensates their decay outside the lens.

Let's explore the requirements to meet the first condition, namely, that of omnidirectional total transmission at the metamaterial interface. The Fresnel amplitude reflectance $\left(r_{12}\right)$ for $p$ polarization at the interface is ${ }^{30,31}$

$$
r_{12}=\frac{\varepsilon_{2 x} k_{1 z}-\varepsilon_{1} k_{2 z}}{\varepsilon_{2 x} k_{1 z}+\varepsilon_{1} k_{2 z}} .
$$

Therefore, for total transmission, i.e., $r_{12}=0$, using Eqs. (3) and (4) we get ${ }^{30}$

$$
\left(\varepsilon_{1}^{2}-\varepsilon_{2 x} \varepsilon_{2 z}\right) \sin ^{2} \theta_{1}=\varepsilon_{2 z}\left(\frac{\varepsilon_{1}}{\mu_{1}} \mu_{2 y}-\varepsilon_{2 x}\right),
$$

where $\theta_{1}$ is the angle of incidence. Evidently, for omnidirectional transmission Eq. (6) must be satisfied for every angle $\theta_{1},{ }^{32}$ which leads to the following conditions:

$$
\frac{\varepsilon_{2 x}}{\mu_{2 y}}=\frac{\varepsilon_{1}}{\mu_{1}}, \quad \varepsilon_{2 x} \varepsilon_{2 z}=\varepsilon_{1}^{2} .
$$

Thus, if Eq. (7) is satisfied the metamaterial would be perfectly matched with an isotropic medium with permittivity $\varepsilon_{1}$ and permeability $\mu_{1}$.

We now proceed to search for the conditions imposed by the second of the listed requirements. We stress that since we deal with anisotropic metamaterial, it is rays of Poynting vectors instead of phase rays that denote the direction of propagation of energy. Therefore, the angles of energy propagation in the RHM (medium 1), $\theta_{S_{1}} \equiv \theta_{1}$, and the metamaterial (medium 2), $\theta_{S_{2}}$, would be

$$
\tan \theta_{S_{1}}=\frac{S_{1 x}}{S_{1 z}}
$$

and

$$
\tan \theta_{S_{2}}=\frac{S_{2 x}}{S_{2 z}}
$$

respectively, with $S_{1}$ and $S_{2}$ being the incident and refracted Poynting vector, respectively. We must ensure that $R_{\theta}$ $=\tan \theta_{S_{2}} / \tan \theta_{S_{1}}$ is negative and independent of $\theta_{S_{1}}$ to satisfy the second of Pendry's perfect-lens conditions. From Eqs. (8) and (9) along with the use of the omnireflectionless condition $r_{12}=0$ and the continuity of the normal component of the displacement vector, $\boldsymbol{D}$, we obtain that

$$
R_{\theta}=\frac{\tan \theta_{S_{2}}}{\tan \theta_{S_{1}}}=\frac{\varepsilon_{1}}{\varepsilon_{2 z}}=\frac{\varepsilon_{2 x}}{\varepsilon_{1}} .
$$

Note, that this simple derivation indicates that the omnireflectionless property simultaneously guarantees the independence of $R_{\theta}$ on $\theta_{1}$. But $R_{\theta}$ must also be negative, mandating $\varepsilon_{2 z}$ and $\varepsilon_{2 x}$ to be negative.

Accordingly, to satisfy the conditions in Eq. (7) $\mu_{2 y}$ must also be negative, i.e.,

$$
\varepsilon_{2 x}<0, \quad \varepsilon_{2 z}<0, \quad \text { and } \mu_{2 y}<0 .
$$

Put it differently, the metamaterial should have an elliptical EFC with an effective metallic character in the $x$ and $z$ direction, and the magnetic resonance excited with a magnetic field along the $y$ direction. Obviously, one-dimensional anisotropic metamaterials ${ }^{27}$ are sufficient to meet such requirements. We stress that our proposed medium is distinctively different from metamaterials with a hyperbolic dispersion. The latter are forward wave media which give only "pseudofocusing" as the far-field source component do not meet at the same focusing point inside the material. ${ }^{17-20}$ Our proposed medium has elliptical dispersion and is backward wave medium.

One can easily confirm the backward nature of the proposed metamaterial on the entire $x z$ plane by checking the product

$$
\boldsymbol{S}_{\mathbf{2}} \cdot \boldsymbol{k}_{\mathbf{2}}=\frac{c}{\omega} \frac{1}{\mu_{2 y}}\left[\left(\boldsymbol{k}_{\mathbf{2}} \times \boldsymbol{E}_{\mathbf{2}}\right)_{y}\right]^{2},
$$

which evidently is negative. Note also that, EM propagation inside the anisotropic metamaterial is extraordinary, with the wave vector, and electric field vector not perpendicular to each other. Therefore, the Poynting vector $\boldsymbol{S}_{\mathbf{2}}$ is not antiparallel to the phase velocity $\boldsymbol{k}_{2}$, as in the case of isotropic left-handed materials but at an obtuse angle with respect to the wave vector. ${ }^{33}$

Using Eqs. (3) and (4) the relation for the incident and refracted angle of the wave vector can be expressed as

$$
\frac{\tan \theta_{k_{2}}}{\tan \theta_{k_{1}}}=\frac{\varepsilon_{1}}{\varepsilon_{2 x}},
$$

where $\theta_{k_{2}}$ is the refracted angle of the wave vector and $\theta_{k_{1}}$ $\equiv \theta_{S_{1}} \equiv \theta_{1}$. Equation (13) implies that the ratio of the incident and refracted angle for the wave vector is independent of the incident angle, as we have found also for the case of the refraction of the energy flow.

Under the reflectionless perfect matching conditions [Eq. (7)], the dispersion relation inside the anisotropic metamaterial takes the simple form

$$
k_{x}^{2}+\frac{k_{2 z}^{2}}{\left(\varepsilon_{2 x}^{2} / \varepsilon_{1}^{2}\right)}=k_{0}^{2} \varepsilon_{1} \mu_{1} .
$$


a)

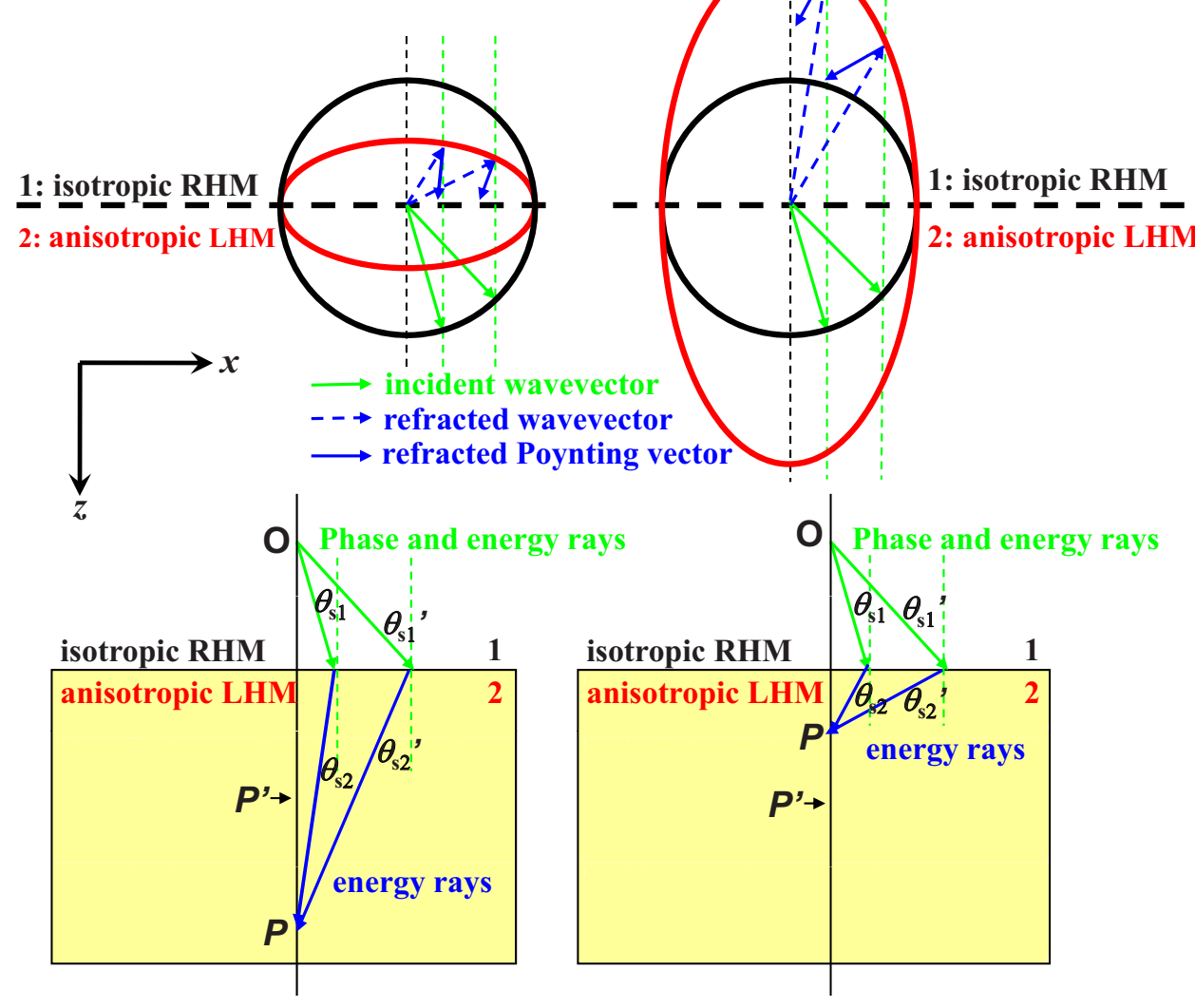

FIG. 2. (Color online) Wave-vector diagram on the $x z$ plane for refraction at the interface between an isotropic regular material, with permittivity $\varepsilon_{1}$ and permeability $\mu_{1}$, and an anisotropic backward metamaterial with $\left|\varepsilon_{2 x}\right|<\varepsilon_{1}$ [case shown in (a)] or $\left|\varepsilon_{2 x}\right|>\varepsilon_{1}$ [case shown in (b)]. The EFCs are shown as circular curves for the isotropic medium and as elliptical curves for the anisotropic metamaterial. Refraction is shown for two angles of incidence (green vectors). The dashed vertical lines are guide to the eyes for the conservation of parallel component of wave vector. The application of all principles governing the refraction give the corresponding refracted wave vectors (dotted blue arrows) and Poynting vectors (solid blue arrows). We observe at the bottom panels the refracted energy rays corresponding to two different arbitrary angles of incidence meet at the same point, $P$, inside the anisotropic metamaterial. For comparison point $P^{\prime}$ is shown which designates the meeting point of two arbitrary beams refracting at the interface of an isotropic metamaterial analog with permittivity $-\varepsilon_{1}$ and permeability $-\mu_{1}$.

Indicatively, we plot the EFCs for the reflectionless anisotropic metamaterial in Fig. 2 (ellipses), along with the EFCs in the isotropic right-handed medium (circles). We feature two cases, which capture all possibilities for any parameter values simultaneously satisfying Eqs. (7) and (11). One with $\left|\varepsilon_{2 x}\right|<\varepsilon_{1}$ shown in panel (a) and one with $\left|\varepsilon_{2 x}\right|>\varepsilon_{1}$ shown in panel (b) of Fig. 2. The conservation of the parallel component of the wave vector expressed in Eq. (2) is depicted for two different arbitrary incident angles with the vertical dotted lines, and determines the refracted wave vectors (dashed arrows). Then the Poynting vectors are the normals at the EFCs at this point and at an obtuse angle with the wave vectors since the medium is backward. We show these as the solid vectors. We note that for each angle of incidence there would have been two distinct choices for a refracted wave vector giving two distinct possibilities for the refracted Poynting vector. We chose here to show only the possibility that is causal, i.e., the one yielding an energy flow that points away from the source. In the bottom panel we transfer the incident and refracted directions of the energy flow in real space. We confirm graphically what Eq. (10), implies, that two incident beams at arbitrary angles cross always at the same point, $P$, inside the metamaterial. For comparison we also show the crossing point, $P^{\prime}$, if the beams were refracting in the isotropic analog of the matched metamaterial. We see that depending on whether $\left|\varepsilon_{2 x}\right|$ is smaller or larger than $\varepsilon_{1}, P$ can lie below or above $P^{\prime}$.

Note that, as expression (14) implies and is graphically seen in Fig. 2, the reflectionless conditions for the metamaterial leads to an elliptical EFC with major axis [case (a)] or minor axis [case (b)] along the interface and equal to the radius of the circular EFC for the medium of incidence. This means that any propagating waves hitting the interface between the right-handed medium and the reflectionless metamaterial will remain a propagating wave inside the metamaterial. Conversely, any evanescent wave hitting the interface between the right-handed medium and the reflectionless metamaterial will remain an evanescent wave inside the metamaterial.

And that brings us to the third requirement of the perfect lens, i.e., the evanescent waves inside the metamaterial should be growing rather than decaying, and thus gain in 
a) Pendry's perfect lens

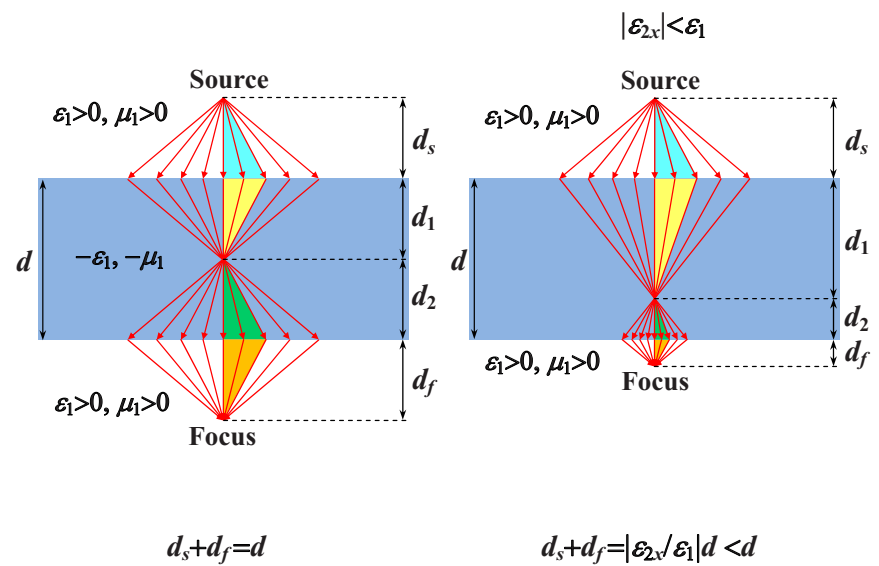

c) Anisotropic perfect lens

$\left|\varepsilon_{2 x}\right|>\varepsilon_{1}$

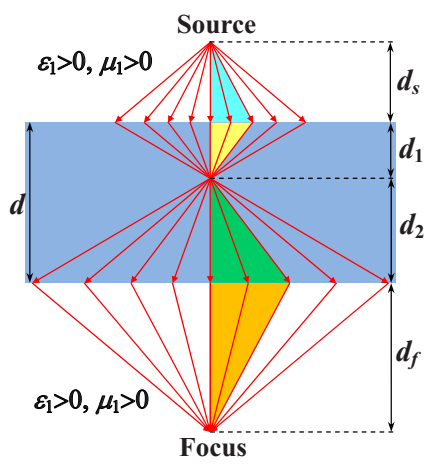

FIG. 3. (Color online) Geometric ray tracing in Pendry's perfect lens [panel (a)], and in anisotropic planar perfect lenses with $\left|\varepsilon_{2 x}\right|$ $<\varepsilon_{1}$ [panel (b)] or $\left|\varepsilon_{2 x}\right|>\varepsilon_{1}$ [panel (c)], respectively. Application of geometry in any of the triangles formed by following the refraction of an arbitrary beam (see, e.g., shaded triangles) yields the lens formula in each case. We show these in the bottom of each respective panel. Note, the arrows represent the energy rays indicating direction of energy velocity not phase velocity.

magnitude while passing through a flat finite slab. This requires the existence of a dispersionless SPP mode. ${ }^{3}$ SPPs for the $p$-polarized case, if present, will be of electric character. $^{34,35}$ The condition to obtain a SPP mode ${ }^{3,35}$ becomes for the case of our reflectionless anisotropic metamaterial

$$
\frac{\sqrt{\left(\beta^{2}-\varepsilon_{1} \mu_{1} k_{0}^{2}\right)}}{\varepsilon_{1}}=-\left|\frac{\varepsilon_{2 x}}{\varepsilon_{1}}\right| \frac{\sqrt{\left(\beta^{2}-\varepsilon_{1} \mu_{1} k_{0}^{2}\right)}}{\varepsilon_{2 x}},
$$

with $\beta=k_{x}$ being the lateral wave vector of the SPPs. It becomes self-evident from Eq. (15) that the SPP condition is met for every $\beta, k_{0}$. In other words, the omnireflectionless anisotropic metamaterial also possesses dispersionless SPPs. So, the evanescent components of the source will increase in magnitude through a slab of thickness $d$ by a factor $M$ (Refs. 1 and 3) where

$$
M=\exp \left(\left|\frac{\varepsilon_{2 x}}{\varepsilon_{1}}\right| \sqrt{\left(\beta^{2}-\varepsilon_{1} \mu_{1} k_{0}^{2}\right)} d\right) .
$$

To summarize, we found that our proposed anisotropic metamaterial lens exhibits on a plane all features of the isotropic perfect lens proposed by Pendry. To realize such lens only a magnetic resonance along one direction is required. This relaxes considerably the metamaterial design requirements to practically realize the perfect lens at the visible frequency regime.

\section{LENS FORMULA FOR THE ANISOTROPIC PERFECT METAMATERIAL LENS}

We have observed in Fig. 2 that any two beams incident at arbitrary angles would meet up at the same point irrespective of the incident angles. This in fact means that all far-field components of a point source would focus at the same point inside the metamaterial slab and then again at the same point outside the lens, just like in the case of Pendry's perfect lens. These type of focusing phenomena allow the derivation of a simple expression which relates the distance between the source and the first interface, $d_{s}$, with the distance between the focus/image and the second interface, $d_{f}$, and the lens slab thickness. Such expression is widely known as the lens formula and is derived simply by geometric considerations and ray tracing of the energy flow.

We show such ray tracing for Pendry's perfect lens and our proposed metamaterial lens in Figs. 3(a)-3(c), respectively. We have distinguished two cases for our proposed metamaterial lens as we have also done in Fig. 2: one with the permittivity component $\varepsilon_{2 x}$ smaller and the other greater than the incoming medium permittivity, $\varepsilon_{1}$, respectively. For Pendry's perfect lens the refracted angle is equal to the incident angle. Geometry in the triangles formed by the refrac-

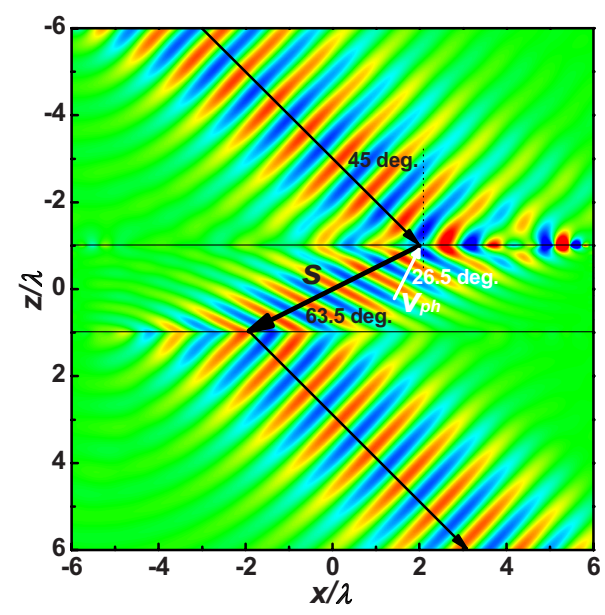

FIG. 4. (Color online) Negative refraction for a beam incident from vacuum at $45^{\circ}$ on a slab made of our proposed anisotropic metamaterial with parameters $\varepsilon_{2 x}=\mu_{2 y}=-2+0.001 i$ and $\varepsilon_{2 z}=-0.5$ $+0.001 i$. Note, the refraction angle of energy flow, $S$, shown with the dark arrow, and the refraction of phase velocity, $v_{\mathrm{ph}}$, shown with the white arrow, are different. 
a) $d s=4 \lambda, d f=6 \lambda$
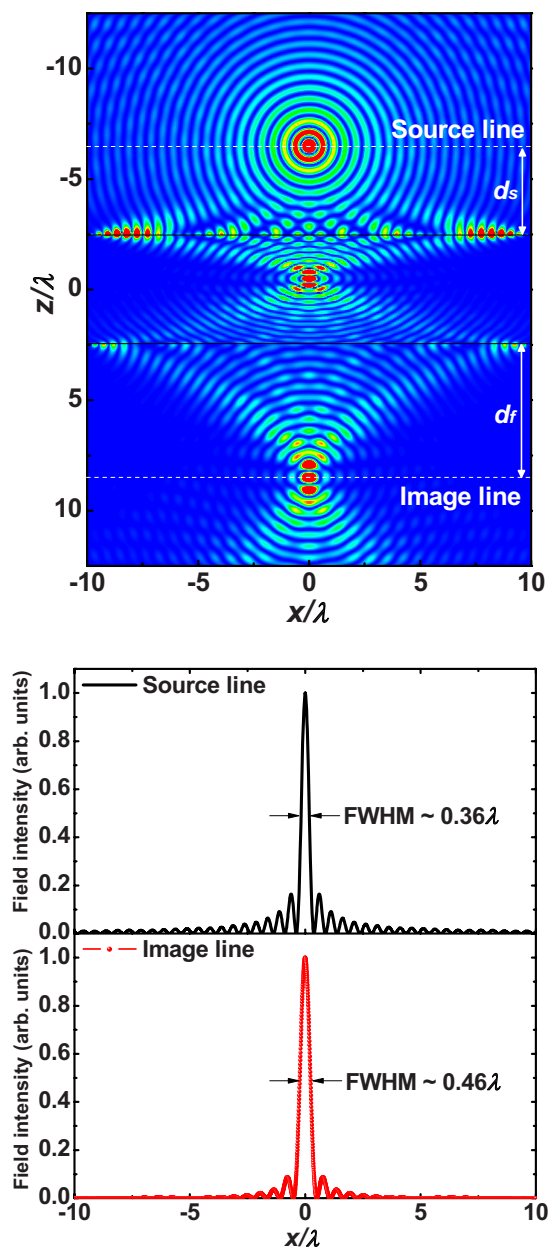

b) $d s=5 \lambda, d f=5 \lambda$
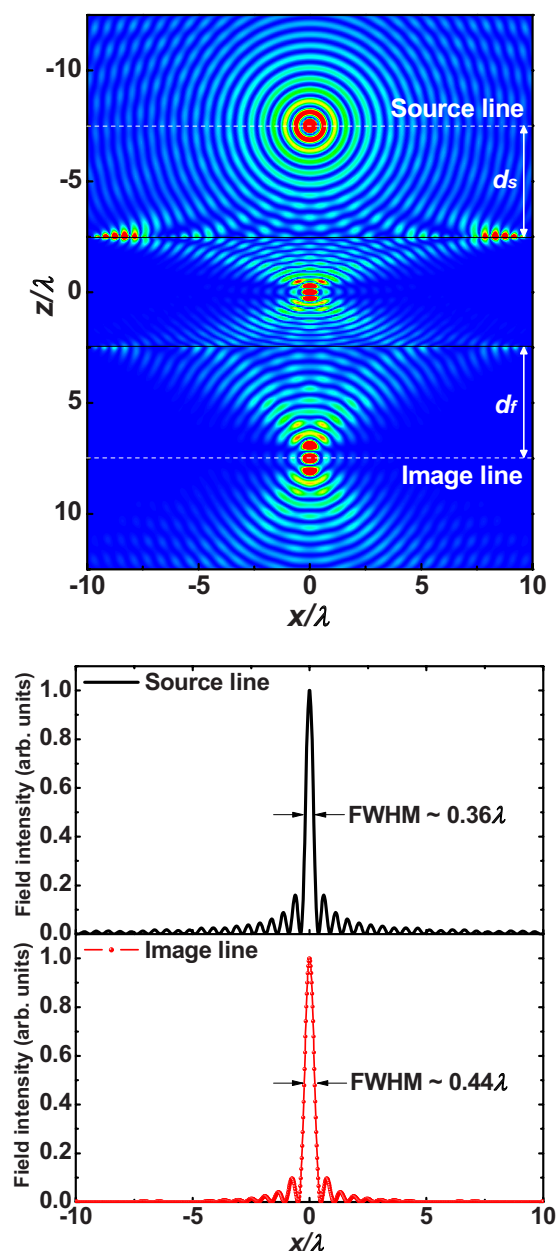

c) $d s=6 \lambda, d f=4 \lambda$
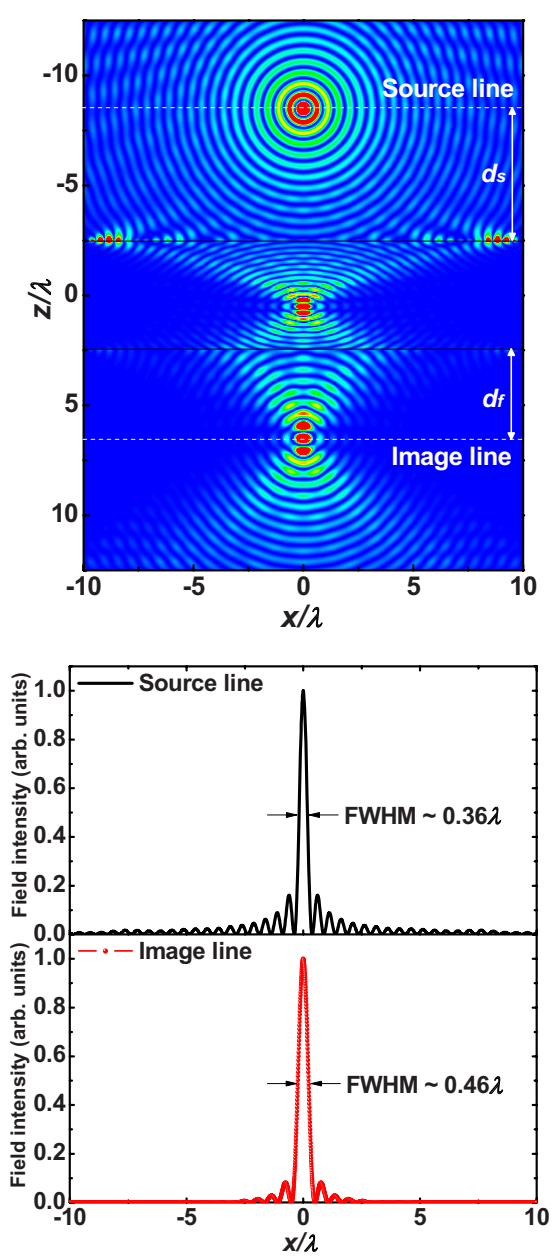

FIG. 5. (Color online) Imaging of a point source through a slab of the anisotropic left-handed metamaterial lens, with parameters $\varepsilon_{2 x}$ $=\mu_{2 y}=-2+0.001 i$ and $\varepsilon_{2 z}=-0.5+0.001 i$, which is perfectly matched to the surrounding vacuum. We consider different distances between the source and the lens in the far-field zone, as given in each respective panel. The solid horizontal dark lines denote the lens limits while the dotted white lines the source and image lines. We can observe a clear focus with location that follows the theoretically predicted lens formula. In the bottom panels we show the respective time-averaged intensities of the magnetic field along the image lines. All FWHM are subdiffraction attesting the superlensing performance of the perfectly matched anisotropic metamaterial.

tion path of an arbitrary beam (see shaded triangles) gives that $d_{1}=d_{s}$, and, $d_{2}=d_{f}$, where the distances $d_{1}$ and $d_{2}$ are defined in the figure. Then this leads to $d_{s}+d_{f}=d$, which is nothing but the long-familiar perfect-lens formula.

We proceed now to derive an analogous formula for the anisotropic metamaterial lens. From geometry in the shaded triangles and using Eq. (10), we get that

$$
d_{1}=\left|\frac{\varepsilon_{1}}{\varepsilon_{2 x}}\right| d_{s}
$$

and

$$
d_{2}=\left|\frac{\varepsilon_{1}}{\varepsilon_{2 x}}\right| d_{f},
$$

Equations (17) and (18) together yield

$$
d_{s}+d_{f}=\left|\frac{\varepsilon_{2 x}}{\varepsilon_{1}}\right| d .
$$

Equation (19) manifests a new lens formula for our anisotropic planar perfect lens. If we use the lens formula, it becomes very easy to see that the magnification factor of the evanescent source components given in Eq. (16), exactly compensates their decay outside, as they arrive at the focus.

The lens formula basically puts a restriction on the farfield zone operation of the lens or the minimum slab thickness for a certain zone of operation. In Pendry's perfect lens, we will not obtain a focusing effect if $d_{s}>d$ while in our anisotropic lens we will not obtain a focusing effect if $d_{s}$ $>\left|\varepsilon_{2 x} / \varepsilon_{1}\right| d$. This has serious implications. Depending on whether the ratio $\left|\varepsilon_{2 x} / \varepsilon_{1}\right|$ is smaller [case of Fig. 3(b)] or greater [case of Fig. 3(c)] than 1, we obtain a more restrictive or a superior lens to Pendry's lens as far as the far-field zone of operation is concerned. The new lens formula suggests 

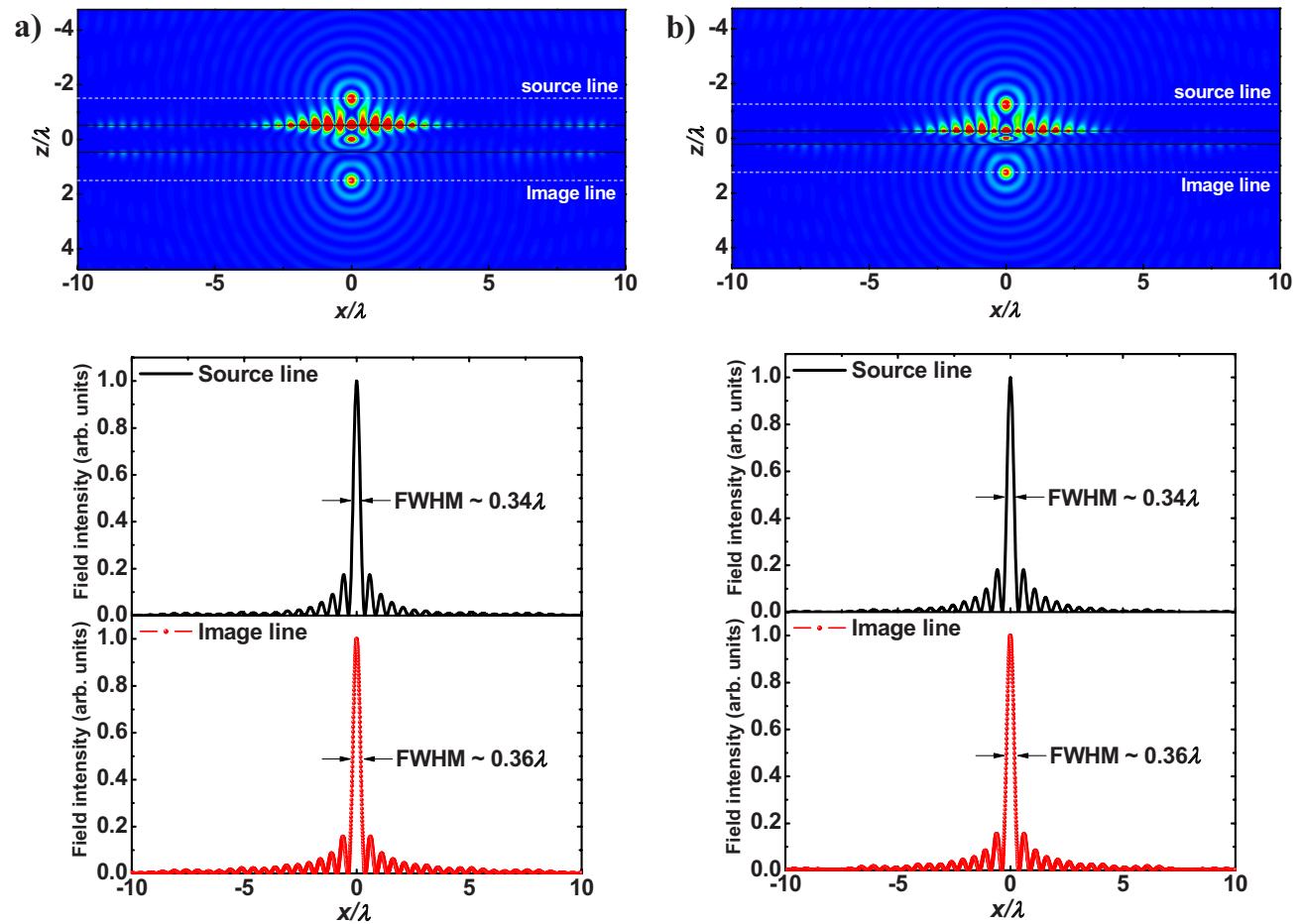

FIG. 6. (Color online) Far-field superlensing with compact anisotropic metamaterial slabs (denoted with the black horizontal lines) perfectly matched to vacuum. The bottom panels depict the time-averaged magnetic field intensities along the image lines (bottom white dotted line in top panel). In both (a) and (b) we have a source placed a wavelength far from the lens and an $0.36 \lambda$ sized image formed one wavelength from the lens, in agreement with the lens formula. In (a) we have considered a slab one wavelength thick made from an anisotropic metamaterial with $\varepsilon_{2 x}=\mu_{2 y}=-2+0.001 i$ and $\varepsilon_{2 z}=-0.5+0.001 i$. In (b) we have considered a slab half a wavelength thick, made from an anisotropic metamaterial with $\varepsilon_{2 x}=\mu_{2 y}=-4+0.001 i$ and $\varepsilon_{2 z}=-0.25+0.001 i$.

that by designing a proper anisotropic metamaterial we can obtain a planar perfect lens, which operates in the far-field zone but is much thinner than its isotropic analog. This is a great advance, as realistic metamaterials are unavoidably plagued with losses that degrade the image intensity.

In the following, we will focus only on the second type of anisotropic metamaterial lens which can be much thinner than the isotropic analog while maintaining a far-field zone of operation. In our theoretical analysis thus far we discussed the complete perfect-lens phenomenon which composes of complete reconstruction of all, radiative and dark, components of the source. However, in practical situation when the near-field components decay substantially, they are at noise level and cannot be compensated anymore by the metamaterial slab. This means that at the far-field zone, the focusing effect practically emanates by the reconstruction of the radiative components of the source only. In the subsequent section we put our proposed lens to test with numerical simulation and compare its performance with the isotropic perfect lens.

\section{NUMERICAL OBSERVATION OF SUPERFOCUSING WITH THE ANISOTROPIC METAMATERIAL LENS}

To test the performance of our proposed anisotropic metamaterial lens, we will consider a metamaterial with $\varepsilon_{2 x}$ $=\mu_{2 y}=-2+0.001 i$ and $\varepsilon_{2 z}=-0.5+0.001 i,{ }^{36}$ which satisfy the anisotropic perfect-lens conditions of Eqs. (7) and (11), em- bedded in vacuum. Realistic metamaterials are always dispersive. This means that in practical situations the perfect anisotropic lens conditions can be met at one frequency only. However, the same is true for Pendry's perfect lens and for many of the reported thus far superlenses. In the following simulations (by COMSOL Multiphysics 3.3), we do not assume any specific frequency of the EM beam but we are scaling both the lateral size and thickness of the metamaterial slab with the free-space wavelength, $\lambda$.

Figure 4 shows the magnetic field of a beam hitting the metamaterial at oblique incidence of $45^{\circ}$. We see that the EM energy of the beam refracts negatively at $63.5^{\circ}$ (dark solid arrow) with the surface normal, which agrees excellent with the predicted value of Eq. (10). As we expected from theory, the wave vector, and accordingly the phase velocity, will not be aligned with the energy flow inside the metamaterial. We depict the phase velocity with the white arrow, which we determined by taking the normal to the phase fronts. The magnitude of the angle between the phase velocity and the surface normal is $\sim 26.5^{\circ}$ and agrees also very well with the predicted value of Eq. (13).

We will use simulations to show that indeed our proposed metamaterial exhibits a far-field superlensing phenomenon subject to a geometric optics lens formula, as expressed in Eq. (19). We consider a metamaterial slab with thickness, $d$, equal to $5 \lambda$, and lateral width equal to $20 \lambda$ and we place a pointlike source at various distances, $d_{s}$, from the first interface. We plot the time-averaged magnetic field intensity in the top panel of Fig. 5(a) for $d_{s}=4 \lambda$, of Fig. 5(b) for $d_{s}$ 

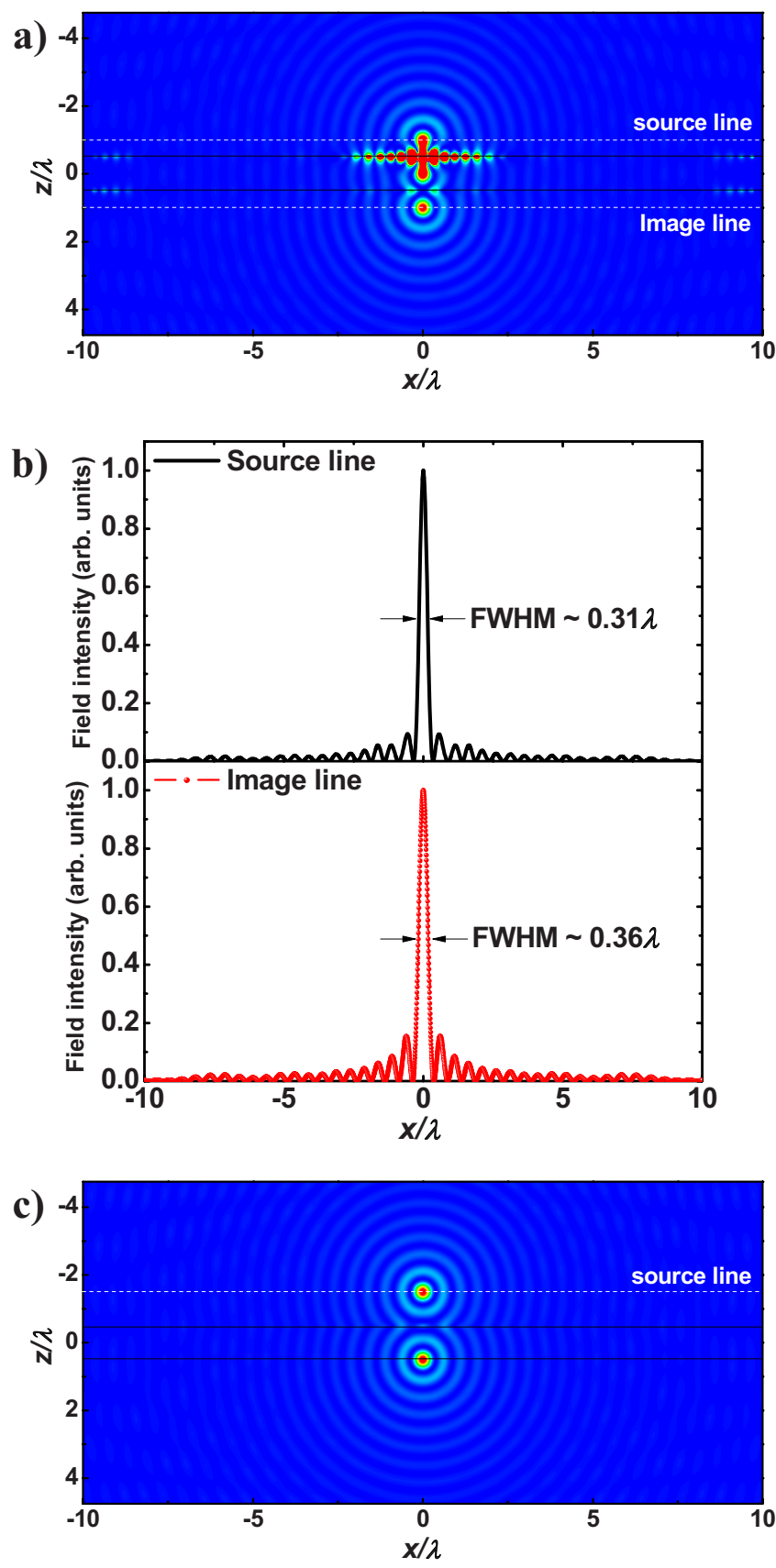

FIG. 7. (Color online) Superfocusing with Pendry's perfect lens analog to the anisotropic metamaterial lens of Fig. 6(a). The lens has isotropic permittivity and permeability both equal to -1 $+0.001 i$, and is one wavelength thick, just like in the case of Fig. 6(a). When we place a source half a wavelength from the lens we obtain an image size of $0.36 \lambda$ [see panel (b), showing magnetic field intensity along image line]. This size is as large as in the case of Fig. 6(a). In panel (c) we see that, as we expected from the perfect-lens formula, we do not get an image behind the lens if we place the source one wavelength away from the lens. (We see the beams meet up exactly on the second interface represented by the bottom black horizontal line.)

$=5 \lambda$, and of Fig. 5(c) for $d_{s}=6 \lambda$, respectively. We see in all cases the clear formation of a focus at a certain distance from the second interface, $d_{f}$. We measured $d_{f}$ to be $6 \lambda, 5 \lambda$, and $4 \lambda$ in case (a), (b), and (c), respectively. So, in all cases $d_{f}$ $+d_{s}=10 \lambda=2 d$, which agrees with the result predicted from lens formula (19), for our choice of parameters. In other words, Fig. 5 attests the validity of the geometric lens formula for our proposed anisotropic superlens.

We look now into the time-averaged intensity in the source and image lines, seen as dashed white lines in the top panel of Fig. 5, and depict these in the respective bottom panels. We have estimated the full width at half maximum (FWHM) in all the cases and show these inside the respective figures. We find image sizes with FWHM that are smaller than half the free-space wavelength, $\lambda / 2$. In other words, we have shown that the anisotropic superlens can operate in the far-field and exhibit subdiffraction focusing capacity.

We also observe in Fig. 5 that the image spot size for case (b) is a little smaller than in cases (a) and (c). If we place now a point source at $d_{s}=8 \lambda$ we find that we continue to see focusing, which still obeys the lens formula, but the image spot size becomes a little larger than $\lambda / 2$. If we place the source more close at $d_{s}=2 \lambda$, which leads at an image formation at $d_{s}=8 \lambda$, we observe again a spot size that is a little larger than $\lambda / 2$. To summarize, our superlens demonstrates subdiffraction focusing in the far-field zone as long as both image and source stay within $\sim 5 \lambda$ from the lens. For larger distances, the focusing still exists but its quality degrades. We attribute this effect to the finite lateral size of the lens, ${ }^{37}$ as for larger source-lens distances the higher spatial frequencies of the source would not hit the interface. As an evidence, we have performed simulations for $d_{s}=5 \lambda$ with different lateral sizes of lens $l_{s}$; the spot sizes (FWHM values) of image become to $0.50 \lambda$ and $0.62 \lambda$ for $l_{s}$ being $15 \lambda$ and $10 \lambda$, respectively.

A hindrance in the practical realization of metamaterial superlenses is loss, which substantially diminishes the intensity of the image. Therefore, it is important to also investigate if it would be possible to obtain a superfocusing effect with thin metamaterial slabs while remaining at least in the close far-field zone on the order of the free-space wavelength. We use now a thinner slab of the same material as in Fig. 5 but with thickness $d=\lambda$ and place a source at distance $d_{s}=\lambda$. Our results, shown in Fig. 6(a), indicate a clear image with a FWHM equal to $0.36 \lambda$, almost as small as the FWHM of the intensity at the source line. Now, let's make the material parameters to be $\varepsilon_{2 x}=\mu_{2 y}=-4+0.001 i$ and $\varepsilon_{2 z}=-0.25$ $+0.001 i$, which also meet Eqs. (7) and (11). Then, the lens formula becomes $d_{s}+d_{f}=4 d$, which allows us to shrink even further the anisotropic metamaterial superlens. We examine this possibility in Fig. 6(b) where we consider a slab made of this material with thickness equal to half a wavelength. Remarkably, even for such a thin lens and a source placed a wavelength away, we observe again subdiffraction focusing obeying the lens formula. This result gives great promise for new interesting metamaterial designs that can lead to the realization of thin planar subdiffraction lenses at visible frequencies.

Finally, we would like to practically compare the performance of our proposed superlens with that of Pendry's perfect lens. We therefore present in Fig. 7 a simulation of the superfocusing capacity of a perfect-lens slab, with thickness equal to one vacuum wavelength and permittivity and per- 
meability equal to -1 . In Fig. 7(a) the source is placed half a wavelength from the lens and we find [see Fig. 7(b)] the formation of an image with FWHM equal to $0.36 \lambda$. This is the same as the case of Fig. 6(a) for the anisotropic metamaterial superlens. The reason we have put the source closer in the perfect-lens slab is the following. If we had put the source one wavelength from the lens as in Fig. 6(a), the focus is exactly on the second interface of the lens, and we find no image behind the lens, as we can observe in Fig. 7(c).

\section{CONCLUSION}

We have shown that a properly designed anisotropic metamaterial can imitate Pendry's perfect lens on a plane. We have theoretically derived all the conditions that a metamaterial has to obey in order to possess, at least on a plane, all of the "key ingredients" of the perfect lens. We have presented simulations that support our theoretical predictions for subdiffraction focusing. The latter obeys a new geometric lens formula which allows for shrinkage of the lens thickness. In particular, our numerical simulations dem- onstrated superfocusing for a lens as thin as half a wavelength, and with an image spot size comparable to that produced by the perfect lens. Our proposal considerably relaxes the stringent mandates of the perfect lens for full isotropy so that offers great flexibility in design and fabrication of superlenses. In addition, the compact superlens is found to be a great promise for practical superlens at the visible according to the state-of-the-art metamaterial fabrication in infrared and optical regimes.

\section{ACKNOWLEDGMENTS}

Authors would like to acknowledge financial support by EU under the projects PHOME (FET Contract No. 213390), ENSEMBLE (NMP-STREP), and ECONAM (NMP-CA), and the COST Actions MP0702 and MP0803; also by the Air Force Office of Scientific Research, Air Force Material Command, USAF (Grant No. FA8655-07-1-3037). Work at Ames Laboratory was supported by the Department of Energy (Basic Energy Science) under Contract No. DE-ACD2$07 \mathrm{CH} 11358$. *nhshen@iesl.forth.gr

†soukoulis@ameslab.gov

${ }^{1}$ J. B. Pendry, Phys. Rev. Lett. 85, 3966 (2000).

${ }^{2}$ V. G. Veselago, Sov. Phys. Usp. 10, 509 (1968).

${ }^{3}$ S. A. Ramakrishna, Rep. Prog. Phys. 68, 449 (2005).

${ }^{4}$ C. M. Soukoulis, J. F. Zhou, T. Koschny, M. Kafesaki, and E. N. Economou, J. Phys.: Condens. Matter 20, 304217 (2008).

${ }^{5}$ C. M. Soukoulis, S. Linden, and M. Wegener, Science 315, 47 (2007).

${ }^{6}$ V. M. Shalaev, Nat. Photonics 1, 41 (2007).

${ }^{7}$ G. Dolling, M. Wegener, C. M. Soukoulis, and S. Linden, Opt. Lett. 32, 53 (2007).

${ }^{8}$ D. Ö. Güney, T. Koschny, M. Kafesaki, and C. M. Soukoulis, Opt. Lett. 34, 506 (2009).

${ }^{9}$ M. S. Rill, C. Plet, M. Thiel, I. Staude, G. von Freymann, S. Linden, and M. Wegener, Nature Mater. 7, 543 (2008).

${ }^{10}$ S. Foteinopoulou, E. N. Economou, and C. M. Soukoulis, Phys. Rev. Lett. 90, 107402 (2003).

${ }^{11}$ S. Foteinopoulou and C. M. Soukoulis, Phys. Rev. B 72, 165112 (2005).

${ }^{12}$ R. Moussa, S. Foteinopoulou, L. Zhang, G. Tuttle, K. Guven, E. Ozbay, and C. M. Soukoulis, Phys. Rev. B 71, 085106 (2005).

${ }^{13}$ X. Wang, Z. F. Ren, and K. Kempa, Opt. Express 12, 2919 (2004).

${ }^{14}$ P. G. Kik, S. A. Maier, and H. A. Atwater, Phys. Rev. B 69, 045418 (2004).

${ }^{15}$ N. Fang, H. Lee, C. Sun, and X. Zhang, Science 308, 534 (2005).

${ }^{16}$ D. Melville and R. Blaikie, Opt. Express 13, 2127 (2005).

${ }^{17}$ Y. Liu, G. Bartal, and X. Zhang, Opt. Express 16, 15439 (2008).

${ }^{18}$ A. Fang, Th. Koschny, and C. M. Soukoulis, Phys. Rev. B 79, 245127 (2009).

${ }^{19}$ Q. Cheng, R. Liu, J. J. Mock, T. J. Cui, and D. R. Smith, Phys. Rev. B 78, 121102(R) (2008).
${ }^{20}$ D. R. Smith, D. Schurig, J. J. Mock, P. Kolinko, and P. Rye, Appl. Phys. Lett. 84, 2244 (2004).

${ }^{21}$ A. Salandrino and N. Engheta, Phys. Rev. B 74, 075103 (2006).

${ }^{22}$ Z. Liu, H. Lee, Y. Xiong, C. Sun, and X. Zhang, Science 315, 1686 (2007).

${ }^{23}$ I. I. Smolyaninov, Y.-J. Hung, and C. C. Davis, Science 315, 1699 (2007).

${ }^{24}$ Z. Jacob, L. V. Alekseyev, and E. Narimanov, Opt. Express 14, 8247 (2006).

${ }^{25}$ K. Aydin, I. Bulu, and E. Ozbay, Appl. Phys. Lett. 90, 254102 (2007).

${ }^{26}$ E. Ozbay, Z. Li, and K. Aydin, J. Phys.: Condens. Matter 20, 304216 (2008).

${ }^{27}$ D. R. Smith and D. Schurig, Phys. Rev. Lett. 90, 077405 (2003).

${ }^{28}$ J. A. Kong, Electromagnetic Wave Theory (EMW, Cambridge, MA, 2005).

${ }^{29}$ L. B. Hu and S. T. Chui, Phys. Rev. B 66, 085108 (2002).

${ }^{30}$ N.-H. Shen, Q. Wang, J. Chen, Y.-X. Fan, J. Ding, H.-T. Wang, and Y. Tian, J. Opt. Soc. Am. B 23, 904 (2006).

${ }^{31}$ N.-H. Shen, Q. Wang, J. Chen, Y.-X. Fan, J. Ding, H.-T. Wang, Y. Tian, and N.-B. Ming, Phys. Rev. B 72, 153104 (2005).

${ }^{32}$ In this sense, any arbitrary incident angle $\theta_{1}$ is a Brewster's angle.

${ }^{33}$ I. L. Lindell, S. A. Tretyakov, K. I. Nikoskinen, and S. Ilvonen, Microwave Opt. Technol. Lett. 31, 129 (2001).

${ }^{34}$ H. Rather, Surface Plasmons (Springer, Berlin, 1988).

${ }^{35}$ R. Ruppin, Phys. Lett. A 277, 61 (2000).

${ }^{36}$ Small loss factor is included in simulations because the surface modes in lossless cases are so strong that will hurt clarity of the numerical results. We have checked that our theoretical analysis can still be considered hold under low loss.

${ }^{37}$ The lateral size of lenses is taken as $20 \lambda$ in the simulations due to the memory limit of computer. 